\title{
AUS DEN VORWORTEN ZUR ERSTEN UND ZWEITEN RUSSISCHEN AUFLAGE
}

Das vorliegende Buch ist der Darstellung der Theorie des elektromagnetischen Feldes und des Gravitationsfeldes gewidmet. Eine vollständige und logisch zusammenhängende Theorie des elektromagnetischen Feldes schließt die spezielle Relativitätstheorie ein, die daher der Darstellung zugrunde gelegt wird. Den Ausgangspunkt für die Ableitung der Grundgleichungen bilden die Variationsprinzipien, mit deren Hilfe man größte Allgemeinheit, Einheitlichkeit und vor allem Einfachheit in der Behandlung erreichen kann.

Dem allgemeinen Plan unserer Lehrbuchreihe ,Theoretische Physik" entsprechend (zu der dieses Buch gehört), gehen wir in diesem Band nicht auf die Elektrodynamik kontinuierlicher Medien ein, sondern beschränken uns auf die ,mikroskopische“ Elektrodynamik, d. h. auf die Elektrodynamik des Vakuums und punktförmiger Ladungen.

Für das Lesen des Buches ist eine Kenntnis elektromagnetischer Erscheinungen nötig, wie sie etwa innerhalb einer allgemeinen Physik Vorlesung vermittelt wird. Es ist ferner eine gute Kenntnis der Vektoranalysis erforderlich. Nicht vorausgesetzt dagegen wird beim Leser eine Kenntnis der Tensoranalysis, die parallel zur Entwicklung der Theorie des Gravitationsfeldes dargestellt wird.

Moskau, Dezember 1939

Moskau, Juni 1947

L. LANDAU, E. LIFSCHITZ 